Tecnología e Innovación

Tecnology and Innovation

Tecnologia e Inovação
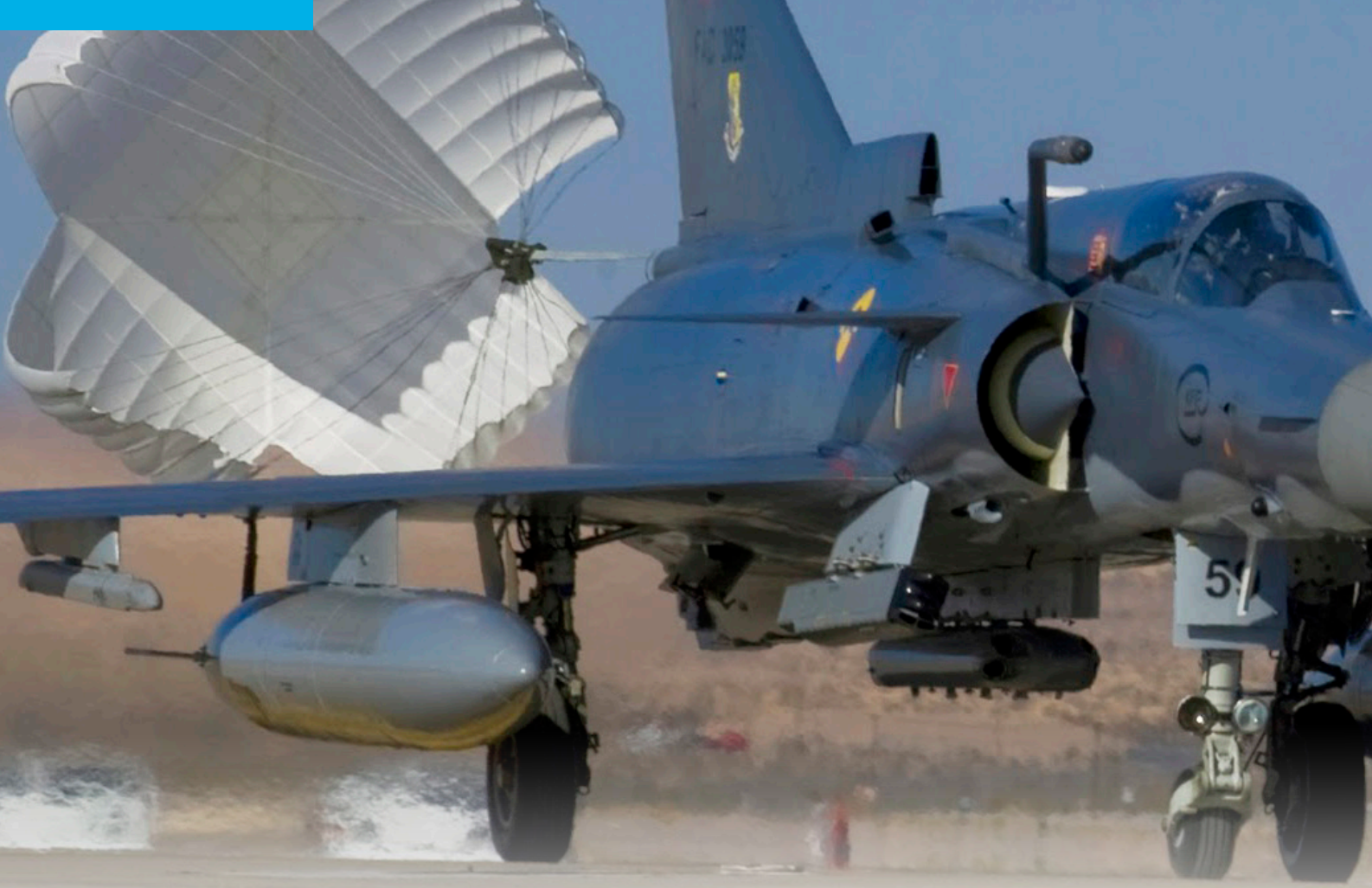

\title{
IMPLEMENTACIÓN DE SISTEMA PARA LA MEDICIÓN DE FUERZAS AERODINÁMICAS EN UN TÚNEL DE VIENTO SUBSÓNICO ${ }^{1}$
}

IMPLEMENTATION OF A SYSTEM FOR MEASUREMENT OF AERODYNAMICS FORCES IN SUBSONIC WIND TUNNEL ${ }^{2}$ IMPLEMENTAÇÃO DO SISTEMA PARA A MEDIÇÃO DE FORÇAS AERODINÂMICAS EM UM TÚNEL DE VENTO SUBSÔNICO ${ }^{3}$

Edgar Leonardo Gómez ${ }^{4}$ Diego Armando Reyes Caballero5 , Julio Enoc Parra Villamil ${ }^{6}$, Johan Steven Galindo López', Eliana Zuluaga ${ }^{8}$

Centro de Estudios Aeronáuticos - CEA / Unidad Administrativa Especial de Aeronáutica Civil. Bogotá, Colombia.

\section{CIENCIA Y PODER AÉREO}

ISSN 1909-7050 / E- ISSN 2389-2468 / Volumen 13 / Número 1 / Enero-Junio de 2018 / Colombia / pp. 106-113 Recibido: 26/02/2018

Aprobado par evaluador: 08/04/2018

Doi: https://doi.org/10.18667/cienciaypoderaereo.590 


\section{Para citar este artículo:}

Gómez, E. L., Reyes Caballero, D. A., Parra Villamil, J. E., Galindo López, J. S. y Zuluaga, E. (2018). Implementación de sistema para la medición de fuerzas aerodinámicas en un túnel de viento subsónico. Ciencia y Poder Aéreo, 13(1), 106-113. doi: https://doi.org/10.18667/ cienciaypoderaereo.590

'Artículo original producto del proyecto de investigación denominado "Desarrollo e implementación de software para la medición de fuerzas aerodinámicas en un túnel del viento subsónico". Financiado y ejecutado por el Centro de Estudios Aeronáutico, con apoyo de pasantes de la Universidad San Buenaventura.

${ }^{2}$ Original article derived from the research project named "Software development and implementation for the measurement of aerodynamic forces in a subsonic wind tunnel", funded and executed by Centro de Estudios Aeronáutico, with the support of Universidad de San Buenaventura interns.

${ }^{3}$ Artigo original produto do projeto de pesquisa denominado "Desenvolvimento e implementação de software para a medição de forças aerodinâmicas em um túnel do vento subsônico". Financiado e executado pelo Centro de Estudos Aeronáuticos, com o apoio de estagiários da Universidade de San Buenaventura.

${ }^{4}$ Coordinación de Investigación, Centro de Estudios Aeronáuticos - CEA / Unidad Administrativa Especial de Aeronáutica Civil. Bogotá, Colombia. Correo electrónico: edgar.gomez@aerocivil.gov.co

${ }^{5}$ Investigador G.IN.A, Centro de Estudios Aeronáuticos - CEA / Unidad Administrativa Especial de Aeronáutica Civil. Bogotá, Colombia. Correo electrónico: diego.reyes@aerocivil.gov.co

${ }^{6}$ Investigador G.IN.A, Centro de Estudios Aeronáuticos - CEA / Unidad Administrativa Especial de Aeronáutica Civil. Bogotá, Colombia. Correo electrónico: julioe.parra@aerocivil.gov.co

${ }^{7}$ Pasante, Centro de Estudios Aeronáuticos - CEA / Unidad Administrativa Especial de Aeronáutica Civil. Bogotá, Colombia. Correo electrónico: johan. galindo@aerocivil.gov.co

${ }^{8}$ Pasante, Centro de Estudios Aeronáuticos - CEA / Unidad Administrativa Especial de Aeronáutica Civil. Bogotá, Colombia. Correo electrónico: eliana. zuluaga@aerocil.gov.co
Resumen: El Centro de Estudios Aeronáuticos (CEA) de la Aeronáutica Civil (UAEAC) ubicado en la ciudad de Bogotá, Colombia, cuenta con un túnel de viento subsónico denominado ELD 402B tipo Eiffel para el desarrollo de prácticas de aerodinámica. Esto le permite al Grupo de Investigación Aeronáutica (G.IN.A) de la institución contar con una herramienta para realizar labores en investigación y docencia. Por lo cual se propone como objetivo desarrollar un sistema de adquisición de datos aerodinámicos y la interfaz del usuario del túnel de viento subsónico. Con el fin de ampliar las capacidades de este túnel de viento, se desarrolló e implementó un sistema que permite adquirir las señales de los sensores de la balanza aerodinámica, ubicados en la sección de pruebas del túnel de viento. La adquisición y procesamiento se realizó con la herramienta de software LabVIEW, con la cual se implementó una interfaz gráfica que permite visualizar las variables entregadas por los sensores, las fuerzas aerodinámicas principales en el perfil alar de prueba, coeficientes aerodinámicos y velocidad relativa del flujo de aire al momento del experimento. En este documento se presentan los resultados de las pruebas de funcionamiento y el proceso de diseño e implementación del sistema que permite hacer mediciones de las variables aerodinámicas, como son los coeficientes de sustentación y arrastre para el modelo que se encuentre en la sección de pruebas del túnel de viento en tiempo real.

Palabras clave: Túnel de viento, balanza aerodinámica, coeficientes de sustentación y arrastre, LabVIEW.

Abstract: The CEA (for Aeronautical Studies Center in Spanish) of the Colombian Civil Aviation Authority (Aerocivil) has a subsonic wind tunnel called ELD 402B Eiffel for the development of aerodynamic practices. This allows to the Aeronautical Research Group to have a tool to carry out research and teaching tasks. The goal of the project presented in this paper, was to develop an aerodynamic data acquisition system and the interfaces for the user. In order to improve the capabilities of this wind tunnel, it was developed and implemented a system that allows to acquire the voltage signals of the aerodynamic balance sensors, located in the test section. The acquisition and processing was carried out with the LabVIEW software tool, used to implement a graphic interface to visualize the variables delivered from the sensors. The variables tested with this tool are, lift and drag coefficients, aerodynamic forces and relative air speed in real time. This paper presents the results of the performance tests, the system design and the implementation process.

Keywords: Wind tunnel, aerodynamic balance, aerodynamic coefficients, LabVIEW.

Resumo: O Centro de Estudos de Aeronáutica (CEA) da Autoridade de Aviação Civil (UAEAC) localizado na cidade de Bogotá-Colômbia, tem um túnel de vento subsônico chamado ELD 402B tipo Eiffel para o desenvolvimento de práticas de aerodinâmica. Isso Ihe permite ao Grupo de Investigação Aeronáutica (G.IN.A) da instituição ter uma ferramenta para fazer o trabalho de pesquisa e ensino. Por isso, propõe-se como objetivo desenvolver um sistema de aquisição de dados aerodinámicos e a interface do usuário do túnel de vento subsônico. A fim de expandir as capacidades deste túnel de vento foi desenvolvido e implementado um sistema que permite adquirir os sinais dos sensores da balança aerodinâmica, localizado na seção de teste do túnel de vento. A aquisição e processamento foi realizada utilizando a ferramenta de software LabVIEW, com o qual implementou-se uma interface gráfica que permite visualizar as variáveis entregues pelos sensores, as forças aerodinâmicas principais no aerofólio de prova, coeficientes aerodinâmicos e velocidade relativa de fluxo de ar no momento do experimento. Neste documento são apresentados os resultados dos testes de desempenho e o processo de design e implementação do sistema que permite fazer medições das variáveis aerodinâmicas, como são os coeficientes de sustentação e arrasto para o modelo que se encontra na seção de provas do túnel de vento em tempo real.

Palavras-chave: DPOC, Internet das Coisas, monitoramento, telemedicina. 


\section{Introducción}

El Centro de Estudios Aeronáuticos (CEA) de la Aeronáutica Civil de Colombia, desarrolla procesos académicos enfocados a estudiantes, docentes, investigadores y personal administrativo; capacita integralmente al personal del sector aeronáutico con el propósito de garantizar la seguridad aérea, el crecimiento de Colombia en términos de investigación, seguridad operacional, fortalecimiento administrativo y el transporte aéreo (Aerocivil, 2016). Consciente de esta política, la UAEAC creó un área de investigación encargada de desarrollar procesos investigativos que generen conocimientos en las áreas propias de la aeronáutica, además de ofrecer soluciones a la industria nacional implementando nuevas tecnologías que permitan el crecimiento del sector aeronáutico.

El túnel de viento es una de las herramientas más utilizadas para estudiar los principios físicos y los conceptos fundamentales de la aerodinámica. La aeronáutica civil cuenta con esta herramienta desde la década de los 90, con el pasar de los años el túnel de viento perdió la capacidad de monitorear las fuerzas aerodinámicas en su sección de prueba. Es por esto que se generó la necesidad de desarrollar un proyecto de investigación que busca mantener en funcionamiento y continua actualización el túnel de viento, ELD 402B tipo Eiffel, con el cual cuenta el CEA.

El objetivo de este proyecto de investigación es el desarrollo de un sistema de adquisición de datos aerodinámicos, como también una interfaz para que los usuarios del túnel de viento obtengan los coeficientes aerodinámicos, valores de fuerza y velocidad del aire en tiempo real. Para el desarrollo de este objetivo se determinan las variables aerodinámicas a trabajar en la sección de pruebas de túnel de viento, posteriormente se desarrolla e implementa el hardware y software de adquisición de datos, y finalmente se desarrolla la interfaz de usuario que permite tener acceso de forma visual a las fuerzas aerodinámicas, los coeficientes aerodinámicos y la velocidad relativa del aire.

\section{Estudios aerodinámicos}

Dentro de los estudios que se desarrollan en el sector aeronáutico, uno de los más representativos es el relacionado con la aerodinámica. Este estudio se aborda generalmente de forma teórica y experimental (Amaya y Betancourt, 2016).

El estudio teórico de la aerodinámica para un perfil alar se caracteriza por determinar matemáticamente las variables físicas obteniendo los coeficientes aerodinámicos. El análisis de los coeficientes aerodinámicos de sustentación y arrastre, sobre un perfil alar, se puede determinar a partir de método CFD (Computational Fluid Dynamics por sus siglas en inglés). Este método permite obtener los coeficientes con la ayuda de las herramientas computacionales y su gran poder de procesamiento matemático (Potts y Masters, 2015).

Dentro del campo experimental se cuenta con herramientas como el túnel de viento, en donde se determinan los valores de coeficientes aerodinámicos.

En el túnel de viento se realizan múltiples estudios de caso y tipos de análisis, como el comportamiento de las distribuciones de presiones sobre cuerpos fuselados, determinando el comportamiento de un perfil alar frente al ángulo de ataque (Meseguer Ruíz, Alonso, Sanz-Andrés, y Pérez-Grande, 2008), entre muchos otros. Los coeficientes aerodinámicos se determinan por varios métodos, sin embargo, el más utilizado y el más recomendado, por su precisión y funcionalidad, es el método en un túnel de viento por medio de una balanza aerodinámica.

\section{Metodología}

Teniendo como fin el desarrollo de un sistema de adquisición de datos aerodinámicos e interfaz del usuario del túnel de viento subsónicos, se implementa un esquema de desarrollo en tres momentos: en el primer momento se definen las variables aerodinámicas a evaluar, una vez se conoce el comportamiento de los perfiles de velocidad, de forma experimental se decide implementar el sensor de transformación diferencial de variación lineal (LVDT), el cual captará los resultantes de la fuerzas aerodinámicas, como son sustentación y arrastre, parametrizando los valores en la sección de prueba del caso de estudio, una vez contrastadas las variables de velocidad y parametrizado el sensor, este se calibra para describir las ecuaciones del modelo matemático, típico al interior de un túnel de viento subsónico; este modelo se implementó en pruebas de flujo y reacción por fuerzas. En el segundo momento se implementa el hardware de adquisición de datos para el túnel de viento subsónico, en conexión con un ordenador. En el tercer momento se desarrolla la interfaz de usuario a través de un software compatible con el ordenador, tipo LabVIEW, que permita al investigador y al usuario, tener acceso a los resultados de fuerzas aerodinámicas y coeficientes correspondientes para el monitoreo de las diferentes pruebas a realizar en el túnel. Obteniendo como resultado el comportamiento de los coeficientes de sustentación y arrastre en la interfaz de usuario.

\section{Túnel de viento}

El caso de estudio presentado en este artículo es el túnel de viento ELD 402B tipo Eiffel del CEA (figura 1). Cuenta con una sección de pruebas de dimensiones: ancho 30.5 $\mathrm{cm}$, largo $53 \mathrm{~cm}$ y alto $30 \mathrm{~cm}$, permitiendo el montaje y pruebas de diversos perfiles aerodinámicos y modelos. 
Estos coeficientes determinan el desempeño de un

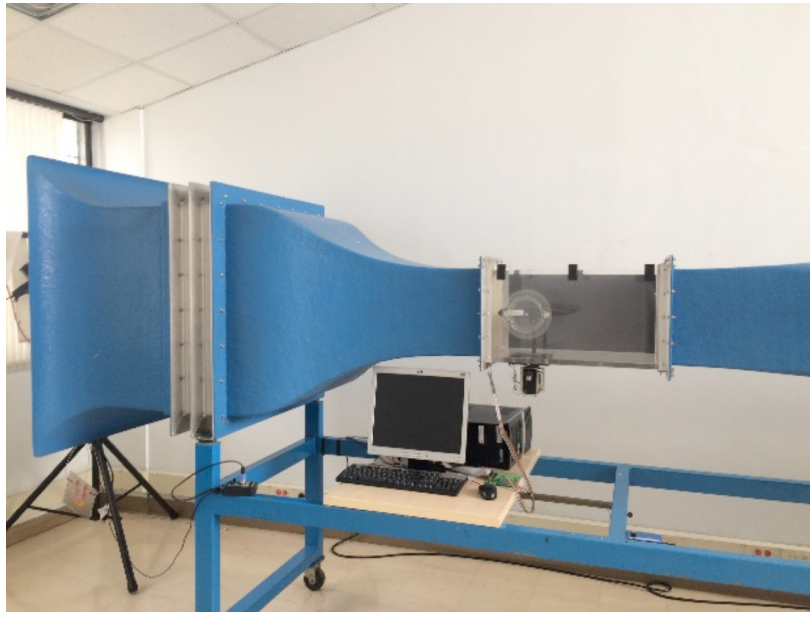

Figura 1. Sección de prueba del túnel de viento Fuente: elaboración propia

El análisis de fuerzas aerodinámicas permite obtener resultados como los coeficientes de sustentación c, (Lift) y coeficiente de arrastre $c_{d}$ (Drag) para un perfil alar o modelo en la sección de pruebas del túnel de viento.

El estudio aerodinámico en túneles de viento depende de varios factores, como la clasificación de flujo compresible e incompresible que generalmente es determinado por un parámetro llamado numero Mach, que relaciona la velocidad del cuerpo con respecto a la velocidad de sonido local (Koreanschi et al. 2017). Este parámetro en túneles de viento, debe determinarse de manera adecuada, ya que la variación de velocidad es un parámetro sensible al momento de determinar las fuerzas aerodinámicas.

La variación de otros parámetros como ángulo de ataque, numero de Reynolds, determinan las gráficas de los coeficientes de sustentación. Los coeficientes aerodinámicos dependen directamente de la fuerza generada por los modelos, la cual es directamente proporcional a la densidad del aire circundante. Por ley de gases ideales, la densidad de un fluido puede ser descrita de la siguiente manera:

$$
\rho=\frac{P}{R T}
$$

En donde P y T son la presión y temperatura del fluido respectivamente, y $R$ equivale a la constante de gases ideales con un valor de $278 \mathrm{~kJ} / \mathrm{kg} \mathrm{K}$.

Es necesario determinar cada una de estas variables al momento de la prueba, con el fin de dar precisión a los coeficientes aerodinámicos obtenidos. perfil alar con respecto a variables mencionadas (Anderson, 2001) de despeje de coeficiente de sustentación en la ecuación (2) y coeficiente de arrastre en la ecuación (3). Como se puede interpretar en las siguientes expresiones:

$$
\begin{aligned}
& C_{1}=\frac{L}{1 / 2 \rho V^{2} C} \\
& C_{1}=\frac{D}{1 / 2 \rho V^{2} C}
\end{aligned}
$$

Donde $\rho$ es el valor de la densidad del fluido al momento de la prueba, $v$ es la velocidad de flujo del aire y $c$ es la cuerda del perfil alar o longitud del modelo. Como se denota en la ecuación (2), los coeficientes son proporcionales directamente a la fuerza, de ahí que es necesario en el laboratorio medir la velocidad, conocer la densidad y principalmente lograr detectar la fuerza vertical y horizontal experimentada por el cuerpo.

El túnel de viento, caso de estudio, permite el montaje y pruebas de diversos perfiles aerodinámicos y modelos, sin embargo, previo al presente trabajo, dicho túnel de viento no contaba con una herramienta que permitiese visualizar los parámetros de fuerza obtenidos en las pruebas. Por lo cual, se desarrolló un sistema de adquisición de datos que permite capturar y representar gráficamente las fuerzas de aerodinámicas generadas en un cuerpo.

Para adquirir los datos y representarlos gráficamente se realiza una programación en el entorno LabVIEW, con un diseño de diagrama de bloques con el fin de transformar las señales de voltaje de los sensores, que perciben la fuerza de sustentación y arrastre en datos gráficos visibles en la interfaz para el usuario.

\section{Balanza aerodinámica}

La balanza es un dispositivo experimental que permite realizar adquisición de datos de fuerzas aerodinámicas que desarrolla un perfil alar durante la prueba (Bahamón, 2014). La balanza, del caso de estudio, cuenta con dos dinamómetros los cuales registran los valores de fuerza aerodinámica. Los dinamómetros deben ser caracterizados bajo una evaluación que permita identificar las señales de salida correspondientes a valores de entrada, obteniendo el comportamiento de las fuerzas.

El dinamómetro es un dispositivo compuesto por dos mecanismos, ubicados en la zona externa de la sección de pruebas para medir las fuerzas de sustentación y arrastre. 
El dispositivo utiliza dos pares de vigas en voladizo, restringidas y separadas por un bloque para cada eje (figura 2). Las fuerzas generadas por el cuerpo en prueba se transmiten al soporte a través de un puntal rígido. Como resultado de la desviación de la viga, la cual es detectada por un LVDT (transformador diferencial de variación lineal), las vigas se desplazan con respecto a la perilla de desviación y el núcleo interno se fija al bloque de la base.

Las señales de voltaje de salida del LVDT son moduladas y amplificadas por los circuitos de acondicionamiento de señal. Los voltajes de corriente directa DC resultantes representan la dirección y magnitud de las fuerzas aplicadas y pueden ser calibrados para lectura de salida en las unidades de cualquier sistema de medidas, es decir; libras de fuerza, Newton, etc.

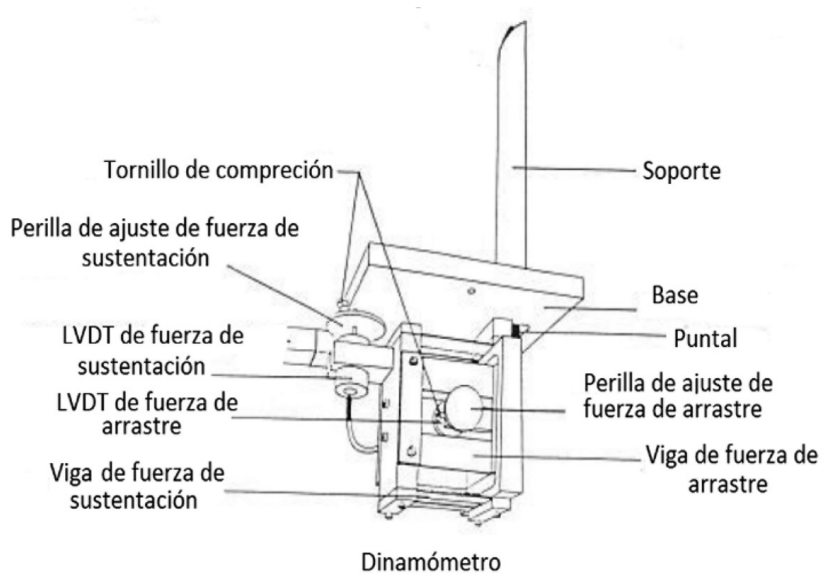

Figura 2. Ensamble dinamómetro

Fuente: manual de operación túnel de viento ELD 402B. Instruction Manual Wing Tunnel Instrumetation System. Engineering Laboratory Desing, Inc. (1990)

\section{Medición experimental}

Se realiza una revisión del estado del arte de los proyectos anteriormente realizados en el caso de estudio, estableciendo un punto de partida para identificar las variables, así como los problemas técnicos del túnel de viento y utilizar las conclusiones de cada una de esas investigaciones para dar una alternativa de solución para la medición de la fuerzas aerodinámicas y velocidad relativa del aire.

El enfoque metodológico se basa en un modelo de adquisición de datos y transducción de las fuerzas aerodinámicas. Las señales son obtenidas a través de los dinamómetros. Se analizan los resultados para diferentes velocidades. El análisis teórico y el desarrollo de la interfaz de usuario dan acceso a las variables de velocidad relativa de viento, fuerza de sustentación y fuerza de arrastre del modelo de prueba.

\section{Resultados}

\section{Caracterización de sensores}

Se caracterizan los dinamómetros implementados con el fin de encontrar un punto óptimo de funcionamiento, adquiriendo una respuesta eficiente de los sensores. La correcta caracterización se basa en el ensamble de dinamómetros encargados de medir la fuerza en la zona de pruebas, funcionando con un rango de frecuencia de entrada de $400 \mathrm{~Hz}$ a $5000 \mathrm{~Hz}$ y un voltaje de entrada de 4 voltios (Vp).

Para determinar valores fiables en las fuerzas aerodinámicas, se evalúa la velocidad de flujo del túnel de viento por medio de un anemómetro; los resultados obtenidos para los sensores de fuerzas y el comportamiento de la velocidad en la zona de pruebas se muestran a continuación (figura 3).

Las fuerzas aerodinámicas se miden en proporción al voltaje generado por los dinamómetros con respecto al desplazamiento de la viga, por efecto de las fuerzas aerodinámicas horizontales para Drag y verticales para Lift y se muestra en las figuras 4 y 5 respectivamente.

Para la medición de las fuerzas de sustentación y de arrastre, se sugiere valores de entrada a los sensores con una frecuencia de entrada de $1000 \mathrm{~Hz}$, ya que presenta un comportamiento lineal para el sensor de fuerza de arrastre y casi lineal para el sensor de fuerza de sustentación, así como poca variación en los resultados al momento de calibrar el sensor. Además, se aplica un voltaje de entrada aproximado a $1 \mathrm{Vp}$ para su correcto funcionamiento.

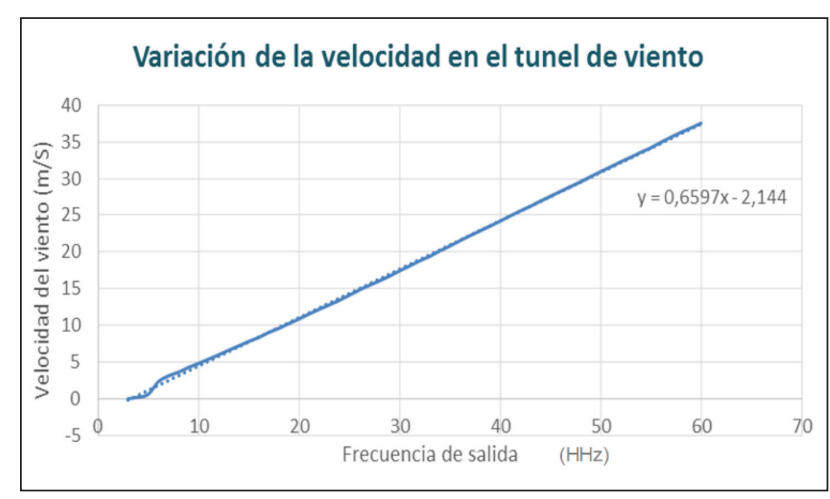

Figura 3. Comportamiento de la velocidad en la zona de pruebas Fuente: elaboración propia 


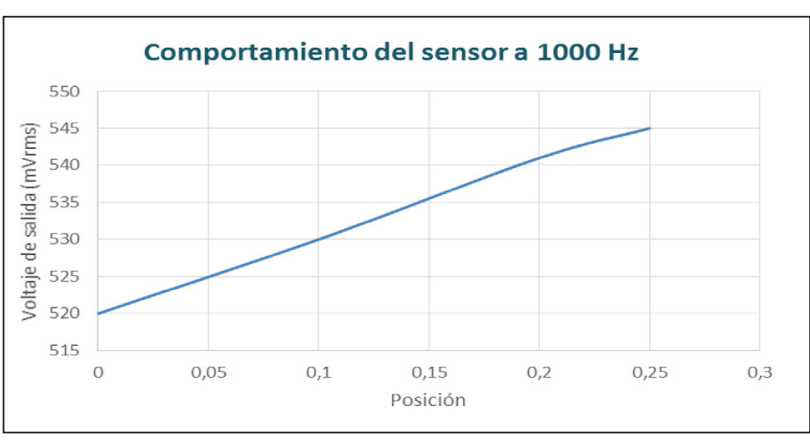

Figura 4. Comportamiento del sensor de fuerza de arrastre Fuente: elaboración propia

\section{Adquisición de datos}

Para capturar las señales de los respectivos sensores se usa una tarjeta de adquisición de datos de National Instruments modelo PCl-6024E, la cual es instalada en la ranura PCMCIA del computador. Para la conexión con los sensores existen dos accesorios, el primero es una tarjeta modelo CB-68-LP con pines de conexión de entradas y salidas según la configuración correspondiente; el segundo es un cable de conexión entre la tarjeta de adquisición de datos y la tarjeta de conexiones modelo R6868.

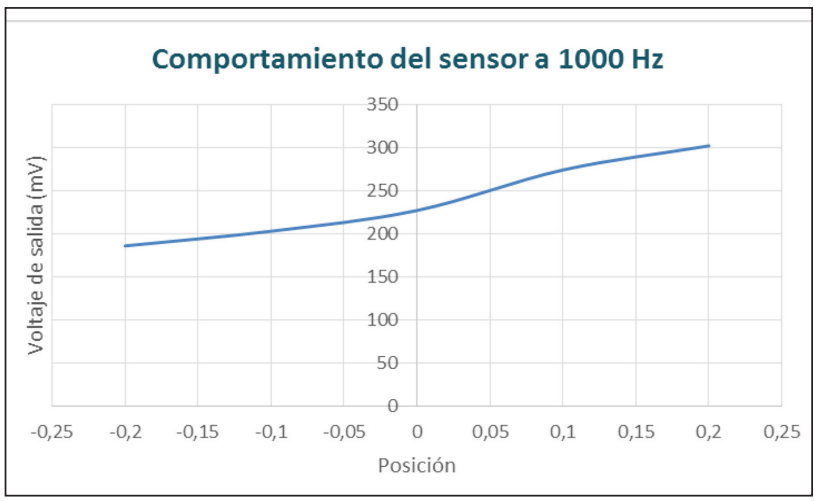

Figura 5. Comportamiento del sensor de fuerza de sustentación Fuente: elaboración propia

En la adquisición de los valores medidos de los componentes externos, el ordenador cuenta con un software llamado MAX (Measurement and Automation Express) que se encarga de realizar la instrumentación virtual, administra los dispositivos conectados al equipo y permite crear herramientas virtuales para la adquisición y desplegado de todas las señales.

Por otro lado, el NI-DAQ es el encargado de seleccionar el controlador adecuado para cada dispositivo conectado, como es el caso de la tarjeta PCI-6024E, que funciona con canales virtuales, estos son registros de tiempo real, a donde van a dar los valores obtenidos de una señal conectada a un canal físico especifico.

\section{Implementación de la interfaz gráfica}

La interfaz gráfica desarrollada es un aplicativo que resume los valores de velocidad relativa equivalente en la sección de prueba, facilita la interpretación de las fuerzas aerodinámicas que desarrollan los modelos en prueba, como se aprecia en la figura 6.

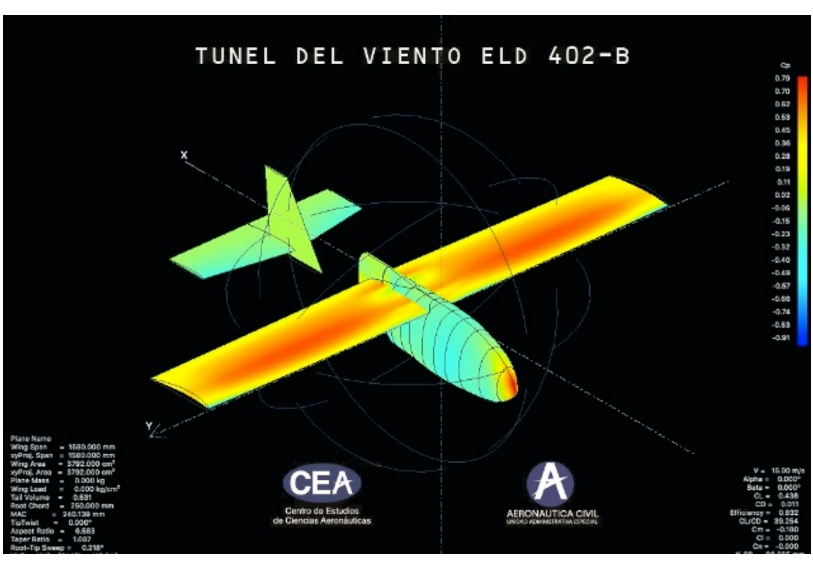

Figura 6. Pantalla principal software Fuente: elaboración propia. XFLR5 v6.03 1/72 (2011)

Para la implementación del sistema de adquisición de datos, caso de estudio, se elabora un instrumento virtual a través de la herramienta LabVIEW, mediante un lenguaje de programación visual. Se crea una plataforma gráfica donde se visualizan las fuerzas de sustentación y arrastre, así mismo la velocidad del flujo del aire equivalente en la sección de pruebas.

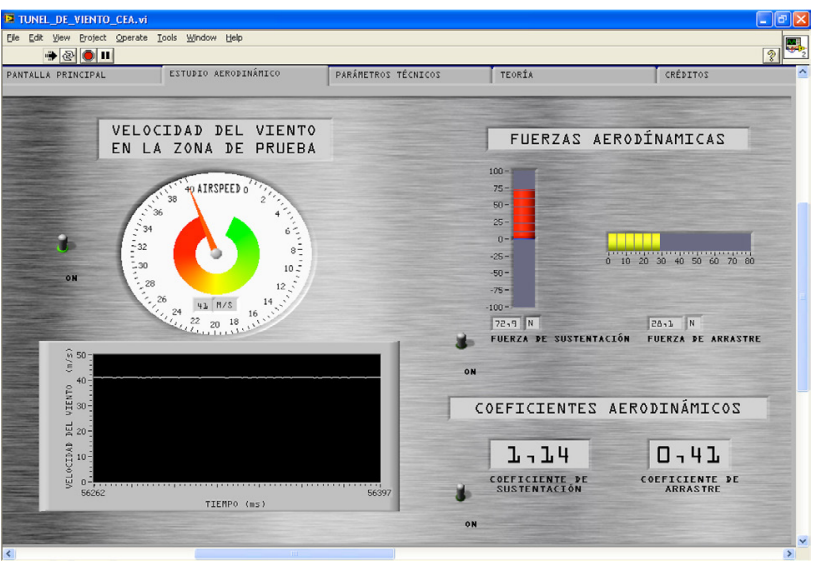

Figura 7. Interfaz en funcionamiento

Fuente: elaboración propia. Labview 8.4 (2011)

\section{LabVIEW}

LabVIEW es una herramienta enfocada en la instrumentación virtual y opera con un sistema de adquisición de datos DAQ (Data Acquisition System), mediante el uso de un ordenador suministrando los recursos necesarios para el análisis, toma de muestras y configuración de los puertos a 
CIENCIA Y PODER AÉREO | ISSN 1909-7050 | E-ISSN 2389-9468 | Vol. 13 | Núm. 1| Ene - Jun 2018 | Escuela de Postgrados de la Fuerza Aérea Colombiana | pp 106-113

utilizar, con programación basada en C (Quiñones, 2011, pp. 115-121).

La programación de la aplicación se realiza por medio de diagramas de bloque, para la generación de la onda de entrada a los sensores se utiliza un módulo DAQ assitant. Este DAQ se configura para generar una onda con las características requeridas para el correcto funcionamiento de cada sensor, con una amplitud y frecuencia determinadas en la caracterización de los sensores, además, para la lectura de las señales de voltaje que son generadas por cada uno de los sensores se utiliza otro DAQ assitant, el cual es configurado para leer la señal y entregar un valor de voltaje correspondiente al cambio que sufre por la fuerza generada sobre el modelo aerodinámico en la zona de pruebas.

Para la transformación de dichas señales en datos teóricos de fuerzas aerodinámicas de sustentación y arrastre, se realizan coeficientes según las ecuaciones previamente citadas. Para ello se utilizan los bloques de aritmética y un bloque denominado fórmula, que permite dar solución a una o varias variables en un sistema de ecuaciones.

En el caso del cálculo de variables como los coeficientes de sustentación y arrastre, se utilizan módulos de aritmética y comparación, para así encontrar dichos valores de forma teórica, por último, todos los valores son representados en forma tal que el usuario pueda analizar el comportamiento dentro de la zona de pruebas, visualizando de forma concreta tanto la magnitud de las fuerzas de sustentación y arrastre, la velocidad del flujo de aire equivalente.

Como resultado de la implementación del sistema, se realizan pruebas exitosas que permiten al Grupo de Investigación del Centro de Estudios Aeronáuticos, contar con una herramienta para el desarrollo de estudios aerodinámicos que se relacionen con la medición de fuerzas aerodinámicas en un túnel de viento.

\section{Factores de error y recomendaciones}

El aplicativo como interfaz de usuario permite ver de manera directa las variaciones de fuerza que se experimentan en un modelo de estudio de manera instantánea, por tal razón los resultados fluctúan constantemente, por la velocidad del sistema adquisidor de datos. Por lo anterior se recomienda presentar los datos con un previo tratamiento, donde se capturen datos por lapsos de tiempo más amplios y se entregue una respuesta promedio.

La balanza aerodinámica está adherida directamente a la sección de pruebas al túnel de viento, razón por la cual las vibraciones mecánicas del túnel son trasmitidas a los dinamómetros, que a su vez afecta la precisión de los datos medidos. Se recomienda soportar la balanza en una estructura independiente al túnel de viento.

La velocidad del túnel es medida a partir de una calibración realizada previamente con un anemómetro, aun así, al ser una variable dependiente del comportamiento de la densidad, presión y temperatura, se recomienda agregar al sistema de adquisición de datos sensores de temperatura y presión para lograr mayor precisión en la velocidad.

\section{Conclusiones}

Al inicio de este proyecto, el Centro de Estudios Aeronáuticos contaba con un túnel de viento operativo con un funcionamiento correcto de forma mecánica, el cual en su sección de prueba se podían observar los fenómenos aerodinámicos, como los son perfiles de flujo laminar y turbulento, pero no se contaba con la metodología para determinar fuerzas que actuaban sobre los modelos en la sección de prueba, como tampoco se poseía una noción de la velocidad del viento que se puede experimentar en la sección de prueba del túnel de viento.

El grupo de investigación consiente de esta necesidad genera un proyecto de investigación, que busca desarrollar un sistema de adquisición de datos aerodinámicos e interfaz del usuario del túnel de viento subsónico, esto para contribuir y desarrollar todas las capacidades del túnel de viento.

En la figura 3 se observan los resultados de velocidad en metros sobre segundo en la sección de pruebas del túnel de viento, a partir de este comportamiento se determina la velocidad relativa de flujo con referencia a la frecuencia de salida del anemómetro. A partir de este comportamiento se predicen los valores de velocidad en la sección de pruebas del túnel de viento.

En la figura 4 se obtienen los valores de voltaje con referencia a la posición del sensor, esta indica los valores de fuerza de arrastre para los modelos de túnel de viento; a partir de la interfaz de usuario se pueden obtener estos valores de fuerza. Al usuario le permite determinar en cada momento los valores de velocidad relativa, fuerzas aerodinámicas y coeficientes aerodinámicos de una forma fácil y organizada.

Como resultado de la implementación del sistema para la medición de fuerzas aerodinámicas en un túnel de viento, es posible concluir que con ayuda de herramientas de software y de hardware se puede desarrollar una solución de instrumentación para representar gráficamente las fuerzas que actúan sobre un perfil alar que está siendo sometido a un flujo de viento en una sección de pruebas, poniendo en uso los recursos necesarios para la adquisición de 
las variables a evaluar, a través de un ambiente interactivo y de fácil manejo, donde claramente se puede realizar un estudio detallado.

Gracias al trabajo realizado el Grupo de Investigación Aeronáutica (G.IN.A) del Centro de Estudios Aeronáuticos, ahora cuenta con una herramienta para el desarrollo de estudios en aerodinámica experimental, contando con la capacidad de determinar fuerzas aerodinámica en los modelos, así como coeficientes de sustentación y arrastre con referencia a la velocidad de flujo.

\section{Referencias}

Aerocivil. Unidad Administrativa Especial de Aeronautica Civil. (2016). Reseña. Laboratorio de investigaciones. Recuperado de https://bit.ly/2HIr7PA

Amaya, O. A. y Betancourt, I. V. (2016). Acondicionamiento de un túnel de viento para pruebas aerodinámicas y prácticas de laboratorio en la UNAH. Revista de Ciencias Especiales, 9(1), 244-263.

Anderson, J. (2001). Fundamentals of Aerodynamics. Recuperado de http://a.moirier.free.fr/A\%E9rodynamique/Bouquins/Anderson/Anderson\%20 \%20Fundamentals\%20of\%20Aerodynamics\%20.pdf
Bahamón, J. J. (2017). Desarrollo de balanza aerodinámica para medición de perfiles alares. (Tesis de maestría). Repositorio Universidad de los Andes. Recuperado de http://repositorio.uniandes. edu.co/xmlui/1992/7783

Meseguer Ruíz, J., Alonso, G., Sanz-Andrés, A. y Pérez-Grande, I. (2008). Algunas consideraciones de carácter docente sobre la circulación en los perfiles aerodinámicos y la posición del punto de remanso anterior. Ingeniería Aeronáutica y Astronáutica, (386), 1-7.

Koreanschi, A., Sugar Gabor, O., Acotto, J., Brianchon, G., Portier, G., Botez, R. M., Mamou, M. y Mebarki, Y. (2017). Optimization and design of an aircraft's morphing wing-tip demonstrator for drag reduction at low speeds, Part II - Experimental validation using Infra-Red transition measurement from Wind Tunnel tests. Chinese Journal of Aeronautics, 30(1), 164-174.

Potts, J. R. y Masters, D. (2015). Validation of the Aerodynamic Loading on Basic Flying Disc Geometries derived from CFD Simulations. Procedia Engineering, 112, 400-405.

Quiñones, C. M. (2011). LabVIEW y la instrumentación virtual aplicados a la docencia y la investigación en ciencias básicas. Revista Elementos, (1), 115-121. 